\title{
Comparison of 3-D Maximum a posteriori and Filtered Backprojection Algorithms for High-Resolution Animal Imaging with microPET
}

\author{
A. Chatziioannou*, Member, IEEE, J. Qi, Member, IEEE, A. Moore, A. Annala, K. Nguyen, R. Leahy, Member, IEEE, \\ and S. R. Cherry, Senior Member, IEEE
}

\begin{abstract}
We have evaluated the performance of two three-dimensional (3-D) reconstruction algorithms with data acquired from microPET, a high resolution tomograph dedicated to small animal imaging. The first was a linear filtered-backprojection algorithm (FBP) with reprojection of the missing data, and the second was a statistical maximum a posteriori probability algorithm (MAP). The two algorithms were evaluated in terms of their resolution performance, both in phantoms and in vivo. Sixty independent realizations of a phantom simulating the brain of a baby monkey were acquired, each containing three million counts. Each of these realizations was reconstructed independently with both algorithms. The ensemble of the 60 reconstructed realizations was used to estimate the standard deviation as a measure of the noise for each reconstruction algorithm. More detail was recovered in the MAP reconstruction without an increase in noise relative to FBP. Studies in a simple cylindrical compartment phantom demonstrated improved recovery of known activity ratios with MAP. Finally, in vivo studies also demonstrated a clear improvement in spatial resolution using the MAP algorithm. The quantitative accuracy of the MAP reconstruction was also evaluated by comparison with autoradiography and direct well counting of tissue samples and was shown to be superior.
\end{abstract}

Index Terms-Image reconstruction, positron emission tomography, small animal imaging.

\section{INTRODUCTION}

$\mathbf{T}$ HE APPLICATION of positron emission tomography (PET) to imaging of small laboratory animals, such as mice and rats requires the highest spatial resolution possible, while still maintaining adequate signal-to-noise ratio in the reconstructed images. We have recently developed an animal PET scanner, microPET, dedicated to high-resolution imaging of small laboratory animals. This tomograph is a fully three-dimensional (3-D) PET system with an intrinsic detector resolution of $1.58-\mathrm{mm}$ full-width at half maximum (FWHM)

Manuscript received September 1, 1999; revised February 3, 2000. This work was supported by the National Cancer Institute under Grants ROI CA69370 and ROI CA59794. The Associate Editors responsible for coordinating the review of this paper and recommending its publication were F. J. Beekman and M. A. Viergever. Asterisk indicates corresponding author.

*A. Chatziioannou, A. Moore, A. Annala, K. Nguyen, and S. R. Cherry are with the Crump Institute for Biological Imaging, Department of Molecular and Medical Pharmacology, UCLA School of Medicine, Los Angeles, CA 90095-1770 USA (e-mail: archatziioann@mednet.ucla.edu).

$\mathrm{J}$. Qi is with the Center for Functional Imaging, Lawrence Berkeley National Lab, Berkeley, CA 94720 USA.

R. Leahy is with the Signal and Image Processing Institute, University of Southern California, Los Angeles, CA 90089-2564 USA.

Publisher Item Identifier S 0278-0062(00)05305-2. and an absolute sensitivity at the center of the field of view (FOV) of $208 \mathrm{cps} / \mu \mathrm{Ci}$. The physical performance characteristics of microPET have been described in detail elsewhere [1]. Traditionally, 3-D image reconstruction is performed with linear algorithms [2]-[4], but the promise of higher resolution and superior noise performance from algorithms that accurately model the system response and the statistical properties of the data have led to the development of 3-D iterative algorithms [5]-[9]. In this work, we compare results obtained using a 3-D filtered backprojection method (FBP), PROMIS, with those obtained using a fully 3-D maximum a posteriori (MAP) method [9]. Studies in simple and complex phantoms and in vivo studies of the rat brain are presented and compared with autoradiography as a gold standard. The main focus to date has been on using the system model to provide resolution recovery with the MAP algorithm [9]. A more detailed analysis of the noise characteristics and relative quantitative accuracy of the MAP reconstruction in comparison with FBP is presented here for both phantom and in vivo studies, and the two reconstruction methods are also compared in terms of absolute quantitation to the widely accepted gold standard of digital autoradiography.

\section{MATERIALS AND METHODS}

\section{A. System Description}

microPET is a high-resolution PET scanner, designed for imaging small laboratory animals [10]. It consists of a ring of 30 position sensitive scintillation detectors, each with an $8 \times 8$ array of $2 \times 2 \times 10$-mm lutetium oxyorthosilicate (LSO) crystals, coupled via optical fibers to a multichannel photomultiplier tube. The detector ring diameter of microPET is $172 \mathrm{~mm}$, with an imaging FOV of $112 \mathrm{~mm}$ transaxially by 18 $\mathrm{mm}$ axially. The scanner has no septa and operates exclusively in 3-D mode, producing 64 sinograms that include all ring differences (0-7) with 100 samples and 120 angles each. The reconstructed image resolution as measured using the 3-D reprojection method [3] is $1.8-\mathrm{mm}$ FWHM isotropically at the center of the FOV.

For both reconstruction algorithms, all ring differences were used, and no approximations, such as angular averaging in the transverse or axial direction, were made. The FBP reconstructions were all performed with a ramp filter cutoff at the Nyquist spatial sampling frequency $\left(0.444 \mathrm{~mm}^{-1}\right)$ and required $10 \mathrm{~min}$ 
for a $128 \times 128 \times 24$ imaging volume using an UltraSPARC 140 workstation with 192 Mbytes of RAM.

The MAP reconstruction algorithm used the factored system model that we developed in [9] to account for photon pair noncolinearity, depth-dependent geometric sensitivities, and intercyrstal scatter and penetration. A preconditioned conjugate gradient algorithm was used to maximize the log posterior density function and differs from that described in [9] only in the use of the shifted-Poisson [11] rather than the true Poisson likelihood. The shifted Poisson model is used to account for the increased variance caused by random subtraction and requires an estimate of the random rate in the sinogram. This was computed for each sinogram from the total number of events in the delayed window by assuming randoms were uniformly distributed after normalization. The prior used in the studies presented here was a Gibbs distribution with a 26-neighbor quadratic energy function.

The smoothing parameter $\beta$ in the prior controls the image resolution/variance tradeoff. For a constant $\beta$, the resolution is count dependent [12]: as the count level increases, the resolution decreases while the variance remains almost unchanged. In contrast, with a fixed filter response in FBP, resolution remains unchanged while variance increases with count level. Of course, in both cases, the increased number of counts leads to an increase in signal-to-noise ratio. By using a count-normalized $\beta$, we can effectively remove dependence of resolution on count rate; i.e., the $\beta$ value selected by the user is normalized by the total number of counts in each frame and this normalized value is used to reconstruct that frame. For the reconstructions shown here, we used a normalized value of $\beta=1.0$ unless stated otherwise and a total of 20 iterations. The reconstruction time was $90 \mathrm{~min}$ for a $128 \times 128 \times 24$ imaging volume on the same workstation.

We note that the $\beta$ normalization described above, and used to process data described in this paper, compensates only for global scaling in the count level; i.e., the resulting image resolution is unaffected by changes in the data acquisition period for a fixed source distribution. The resolution of the image is spatially variant for reasons described in [13]. To compensate for this effect and produce uniform resolution, we can use the spatially variant smoothing method described in [14].

\section{B. 3-D Baby Monkey Phantom}

A small baby monkey brain phantom constructed in an analogous fashion to the 3-D Hoffman brain phantom [15], with an outer diameter of $4 \mathrm{~cm}$ and an active cross section of $3.3 \mathrm{~cm}$ was filled with $0.77 \mathrm{mCi}$ of ${ }^{18} \mathrm{~F}$ and imaged for 120 frames, with $30-\mathrm{s}$ imaging time per frame. To generate data with essentially an equal number of counts, the 120 frames were added in the pairs $1+120,2+119, \ldots, 60+61$, resulting in 60 frames each within $2.5 \%$ of three million counts. A calculated attenuation correction was applied to the data using a $\mu$-value of $0.095 \mathrm{~cm}^{-1}$. Each frame of the 60 realizations was reconstructed with both algorithms, generating an ensemble of 60 independent realizations that allowed an image of the standard deviation to be estimated for the MAP and FBP algorithms [16]. A slice from the phantom was also digitized and blurred to the resolution of the MAP reconstruction and is shown in Fig. 1 for visual comparison with the reconstructed images.

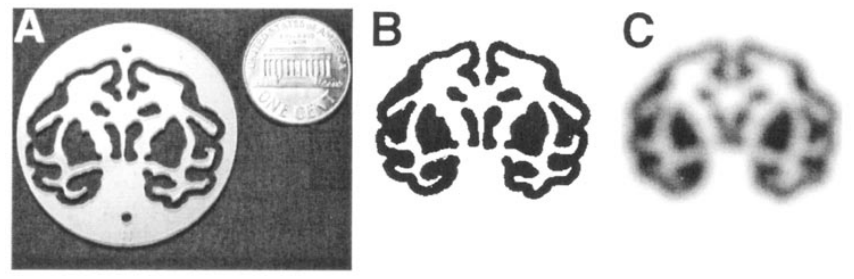

Fig. 1. (A) Picture of a slice from the baby monkey brain phantom, illustrating its design and physical size. (B) Digital image of the same slice. (C) Same slice blurred to approximately match the resolution of the MAP reconstruction.

\section{Recovery and Spillover Comparison}

A cylindrical phantom, measuring $4 \mathrm{~cm}$ in diameter and containing circular cross-section compartments of diameter $3 \mathrm{~mm}, 4$ $\mathrm{mm}$, and $10 \mathrm{~mm}$ was imaged in microPET. The cylinder was 3.5 $\mathrm{cm}$ long and had axial symmetry. The background chamber was filled at a concentration of $8 \mu \mathrm{Ci} / \mathrm{ml}$ with ${ }^{18} \mathrm{~F}$, whereas the circular compartments were filled with ${ }^{13} \mathrm{~N}$ at an initial concentration of $92 \mu \mathrm{Ci} / \mathrm{ml}$. The phantom was scanned dynamically over a 75-min time period, during which time, the compartment to background activity ratio changed from roughly $10: 1$ to $1: 10$. Regions of interest (ROI's), matching the cross section of the circular compartments, were drawn on the MAP and FBP images. Another ROI was drawn over the background and was located such that it would experience insignificant partial volume or spillover effects. The apparent activity ratio between the compartments and background was compared with the known true activity ratio, which was measured in a well counter.

\section{Resolution versus Noise Comparison}

The same cylindrical compartment phantom as in Section C above was filled with a total of $0.5 \mathrm{mCi}^{18} \mathrm{~F}$ and an activity ratio of 4.22:1 (background $=20 \mu \mathrm{Ci} / \mathrm{cc}, 10-\mathrm{mm}$ diameter compartment $=4.74 \mu \mathrm{Ci} / \mathrm{cc}$ ). A data set containing 170 million total counts was acquired and reconstructed with both the FBP and the MAP algorithms. For the MAP reconstructions, 12 different smoothing parameters $\beta$ were used ranging from 0.125 to 16 , producing images with different spatial resolutions. The FBP image was at first reconstructed with a ramp filter and subsequently smoothed with a Gaussian kernel to spatial resolutions ranging from intrinsic $(1.8 \mathrm{~mm})$ to $3.0 \mathrm{~mm}$. In addition, the FBP reconstructed image was deconvolved with linear deconvolution to produce images with higher resolution than the ramp-filtered reconstructions. The resulting resolution of these reconstructed sets of images was estimated by drawing a radial profile from the center of the 10 -mm cold cylindrical region and extending it over the hotter background. These profiles were then correlated to simulated profiles of the same edge with known resolutions and the known contrast from well counter measurements. In addition, ROI's were drawn over the uniform background region, small enough not to include partial volume effects, and the standard deviation in these ROI's was used as an estimate of the noise in the reconstructed images.

\section{E. In Vivo Studies}

A normal Sprague-Dawley rat was injected with $3 \mathrm{mCi}$ of ${ }^{18}$ F-fluorodeoxyglucose (FDG). After a 40-min uptake period, 
the rat was anesthetized and placed in a stereotactic headholder [17] and scanned on microPET for $60 \mathrm{~min}$. Images were reconstructed with FBP (ramp filter) and MAP. A second rat was injected with $2 \mathrm{mCi}$ of FDG. Following the uptake period of $45 \mathrm{~min}$, the rat was sacrificed with an overdose of pentobarbitol to prevent any further redistribution of FDG. The rat was then decapitated, and the head was placed on a bed of dry ice. The whole head was then imaged in the microPET scanner for 30 min. Following scanning, the frozen whole head was packed in dry ice and rapidly sliced in $45-\mu \mathrm{m}$ thick slices. The slices were placed on phosphor storage plates and exposed along with ${ }^{14} \mathrm{C}$ standards for three days. The phosphor plates were subsequently read out using a Fuji BAS 5000 system to produce digital autoradiograms of the distribution of FDG in the rat head for comparison with MAP $(\beta=1.0)$ and FBP microPET images. A calculated attenuation correction was applied to both of these studies. This correction was based on finding the contours of the head [18] and used $\mu$-values of $0.095 \mathrm{~cm}^{-1}$ for tissue and $0.115 \mathrm{~cm}^{-1}$ for bone, assuming a skull thickness of $1 \mathrm{~mm}$. All animal protocols were approved by the UCLA animal research committee.

\section{RESUlTS AND DISCUSSION}

\section{A. 3-D Baby Monkey Phantom}

Figs. 2 and 3 show the results from the 3-D monkey brain phantom. A (FBP) and D (MAP) in Fig. 2 are reference reconstructions of the data summed across all frames (180 million counts). B and $\mathrm{E}$ are reconstructions of individual realizations (three million counts) with FBP and MAP, respectively. C and $\mathrm{F}$ in Fig. 2 are the point-to-point noise images, estimated from the standard deviation of the 60 realization ensemble according to (1), where $S$ is the standard deviation in an image pixel, $m_{i}$ is the value of the image in that pixel in measurement $i, N$ is the total number of measurements, and $\bar{m}$ is the value in that pixel as measured with the reference images

$$
S=\sqrt{\frac{1}{N-1} \sum_{i=0}^{N}\left(m_{i}-\bar{m}\right)^{2}} .
$$

When the image D in Fig. 2 is compared with the images of $\mathrm{B}$ and $\mathrm{C}$ in Fig. 1, it is clear that the additional structures seen in the MAP reconstruction compared with the FBP represent true features of the phantom. In the profiles illustrated in Fig. 3, the MAP reconstruction demonstrates better recovery of structure and smaller partial volume and spillover effects suggestive of higher spatial resolution than does the FBP. To better illustrate the magnitude of the noise levels in the standard deviation images of $\mathrm{C}$ and $\mathrm{F}$ in Fig. 2, Fig. 3 also has horizontal profiles through the images of $\mathrm{C}$ and $\mathrm{F}$ in Fig. 2. In addition, an ROI was drawn over the standard deviation images of $\mathrm{C}$ and $\mathrm{F}$ in Fig. 2, tracing the objects outline. Inside that region, the average standard deviation was 0.486 for the FBP and 0.484 for the MAP reconstructions, indicating that the noise levels are comparable.

In order to evaluate the possible bias caused by variations in the count levels in the two reconstruction algorithms, an ROI was drawn in the cortical area of the monkey brain phantom. The count levels in this ROI were measured for seven added frame
A

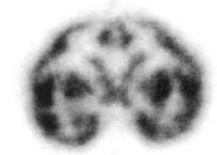

D

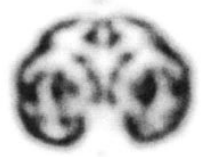

B

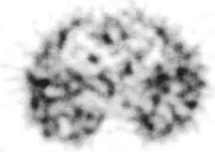

E

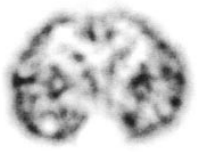

C

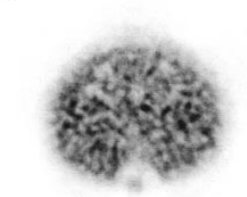

Fig. 2. Images A-C correspond to FBP, whereas images D-F correspond to MAP reconstructions (A). Reconstruction of the summed data set. (B) Reconstruction of a single realization. (C) Image of the standard deviation in reconstructions.

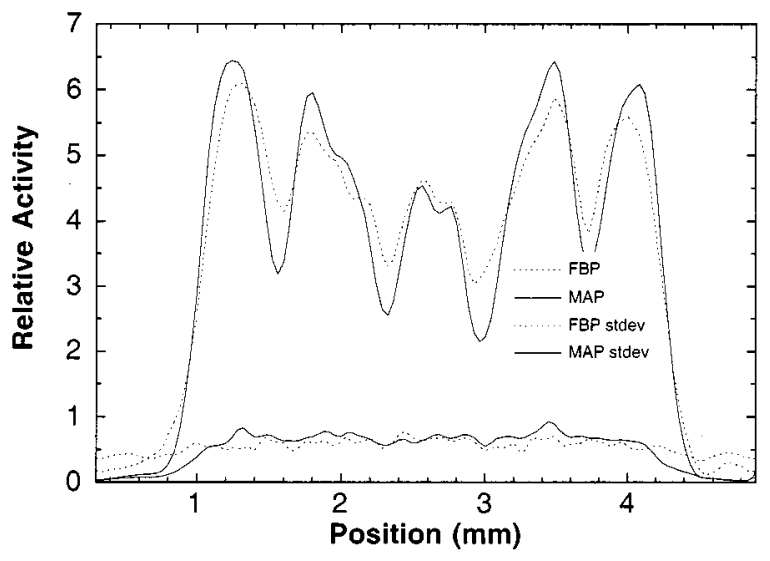

Fig. 3. Horizontal profiles through the summed and standard deviation images of Fig. 2. Notice better recovery and reduced spillover in the profile through the MAP image, suggestive of higher spatial resolution. The standard deviation profiles illustrate that the noise is comparable in the two reconstruction methods.

levels, containing 3, 6, 12, 24, 38, 96, and 180 million counts each. Fig. 4 is a plot of the mean ROI value as a function of the total number of counts in the frame. The ROI values in the MAP reconstructions are consistently higher than is the FBP across all count levels, because of a reduced partial volume effect, indicating improved contrast recovery for the MAP reconstructions. At the same time, the absolute ROI values are constant for both reconstruction algorithms at all frame count levels, which range across almost two orders of magnitude. This is expected from the linear FBP algorithm, but it proves that the MAP reconstruction has virtually no bias caused by variations in count levels.

\section{B. Recovery and Spillover Comparison}

Figs. 5 and 6 show a comparison of the measured activity ratio compared with the true ratio for the different sized compartments in the cylindrical phantom. Notice better recovery when the compartments have higher concentration than in the background (Fig. 5) and less spillover when the compartments have lower concentration than in the background (Fig. 6) for the MAP reconstruction compared with FBP. The partial volume and spillover effects in this experiment are exacerbated because of the finite wall thickness of the compartments in the cylinder 


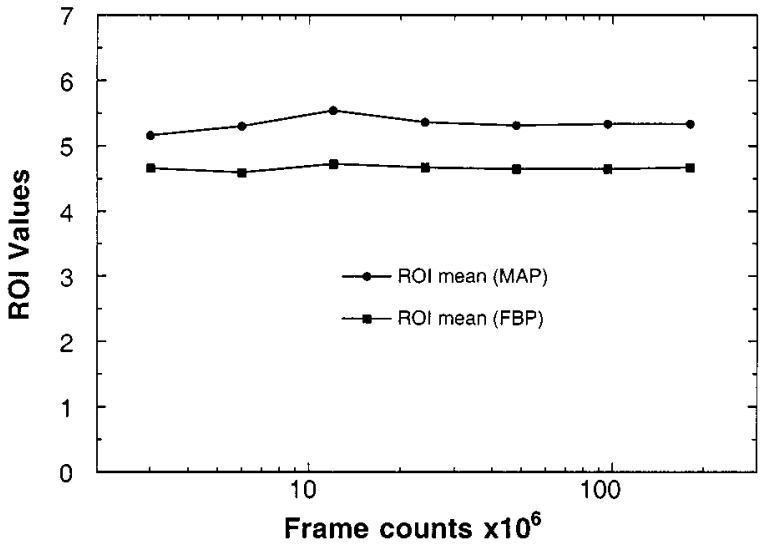

Fig. 4. ROI values in cortical region of the monkey brain phantom, as a function of the total number of counts in the study.

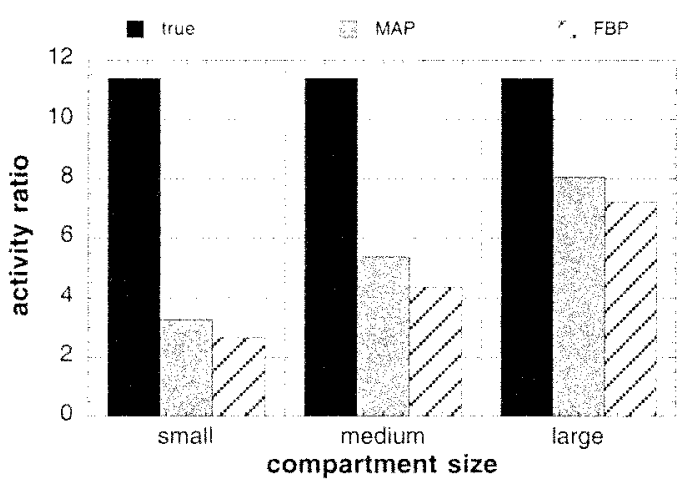

Fig. 5. Comparison of known activity ratio (11.4:1) between compartments and background, to measured ROI ratio. In all cases, MAP reconstructions provide a better estimate of the true activity.

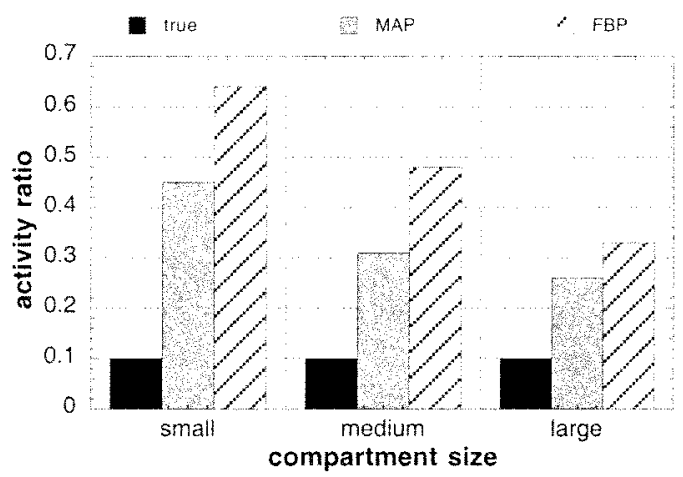

Fig. 6. Comparison of known activity ratio $(1: 10)$ between compartments and background, to measured ROI ratio. In all cases, MAP reconstructions provide a better estimate of the true activity.

and the deliberate choice of large ROI's that are matching the compartment size. This choice highlights the quantitative differences between MAP and FBP. The graphs present strong evidence that the MAP reconstruction is recovering resolution beyond that seen using FBP and a ramp filter.

\section{Resolution versus Noise Comparison}

The image spatial resolution versus noise as measured in the compartment phantom is illustrated in Fig. 7. It is clear from that figure that at all matched noise levels, the MAP reconstructions

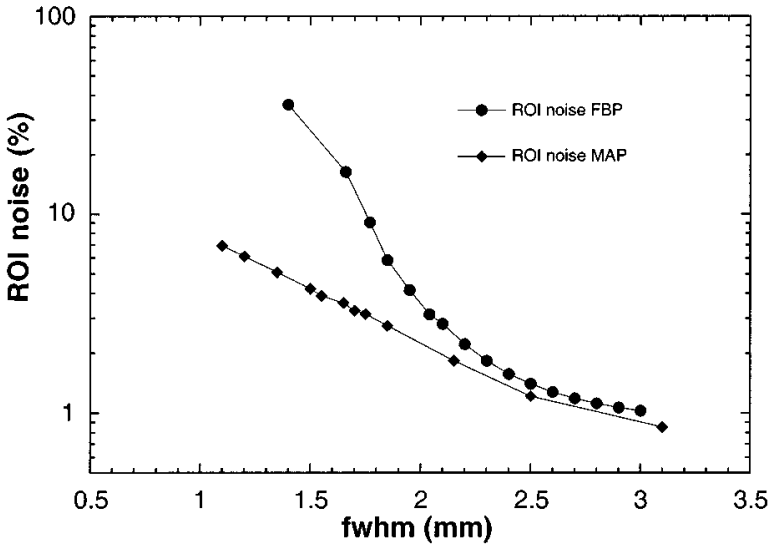

Fig. 7. Measured resolution versus noise curves for MAP and FBP.

have higher spatial resolution. This advantage is especially pronounced at higher spatial resolution levels. As the spatial resolution in the images decreases (FWHM increases), the benefits of the MAP reconstruction become less pronounced. For the FBP reconstruction with a ramp filter, the quantitative improvement in resolution of the MAP reconstruction is $20 \%$ with matched noise levels. As the spatial resolution of both the reconstructed images becomes better, the relative resolution improvement of the MAP reconstruction over FBP becomes more significant for matched noise levels.

\section{In Vivo Studies}

Fig. 8 shows a comparison of coronal slices through MAP and FBP reconstructions in a normal rat brain. The MAP reconstruction clearly demonstrates an improvement in resolution and better delineation of anatomical structures over FBP with a ramp filter. The MAP shows improved separation of cortical and subcortical structures, without an associated increase in the image noise level. Image A in Fig. 9 shows a coronal anatomical slice, selected from the digital whole-head ${ }^{18} \mathrm{FDG}$ autoradiograph, which had an intrinsic spatial resolution of $0.2 \mathrm{~mm}$. B and C in Fig. 9 are MAP and FBP reconstructions, respectively, of the corresponding slice, measured with microPET from the same rat head and the same isotope injection. Again, in those images, the improvement in spatial resolution of MAP over ramp-filtered FBP is clear. In the same figure are also illustrated the ROI's used for the quantitative evaluation of the reconstruction algorithms.

Fig. 10 shows the absolute tissue activity concentration in $\mu \mathrm{Ci} / \mathrm{g}$ measured from ROI's defined over cortical and subcortical structures in the autoradiograph and the reconstructed PET images. In this figure, the cortical regions and the white matter are quantified more accurately with MAP rather than with FBP. The reason that the striatum measurement is inadequately quantified by both reconstruction methods is because of its small physical size and its location next to the cortex.

\section{CONCLUSION}

It is clear from the phantom and in vivo data that MAP reconstruction is able to recover resolution beyond that possible using FBP with a ramp filter. This leads to better visualization of 
MAP
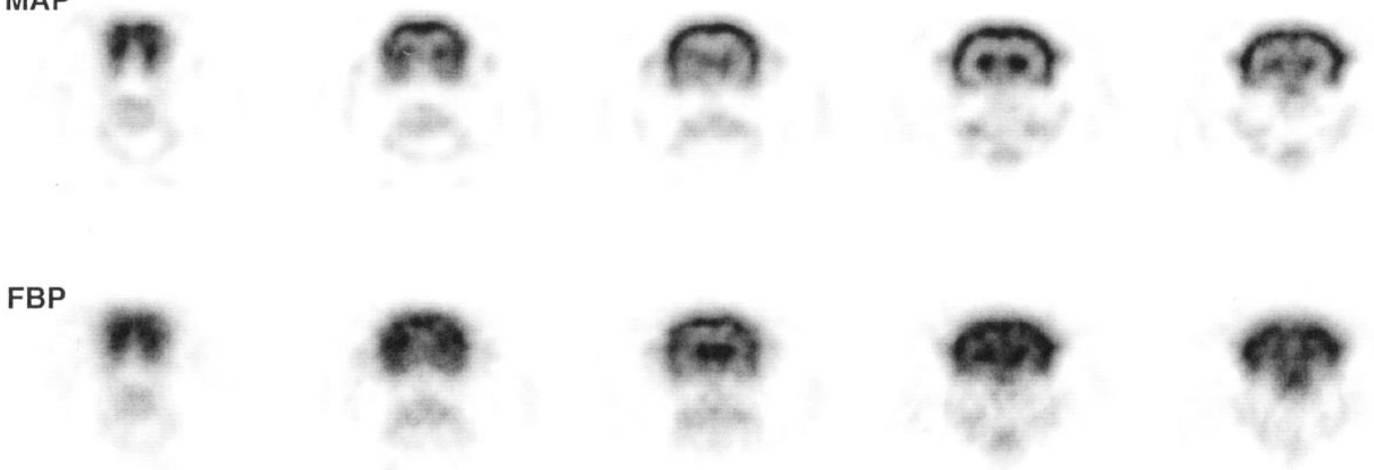

Fig. 8. In vivo coronal images from MAP and FBP reconstructions, illustrating FDG distribution from the front to the back of a normal rat brain. The MAP reconstruction is capable of separating cortical from subcortical structures and clearly shows a significant improvement in spatial resolution, without an increase in noise.
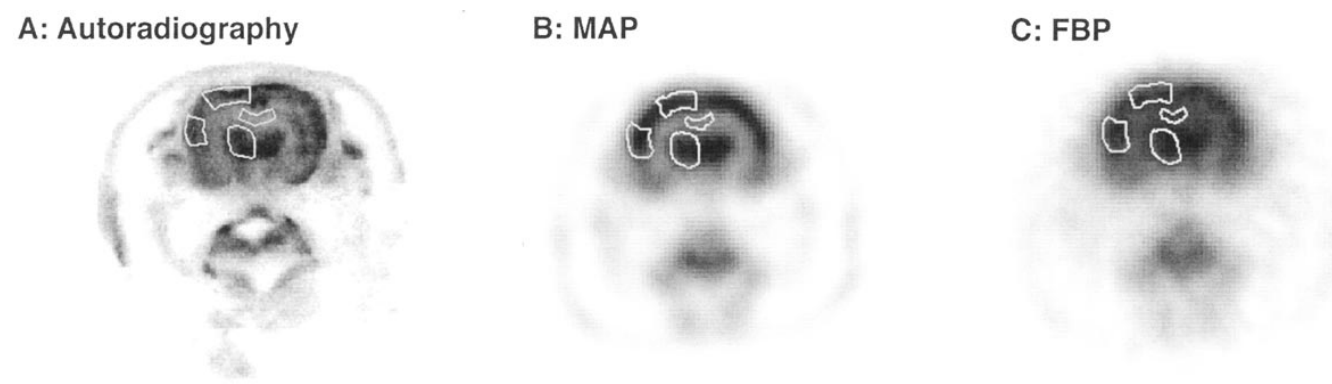

Fig. 9. Coronal slices from digital autoradiography, as well as corresponding microPET images of the same rat reconstructed with MAP and FBP, illustrating the cortical regions where the quantitative ROI's were drawn.

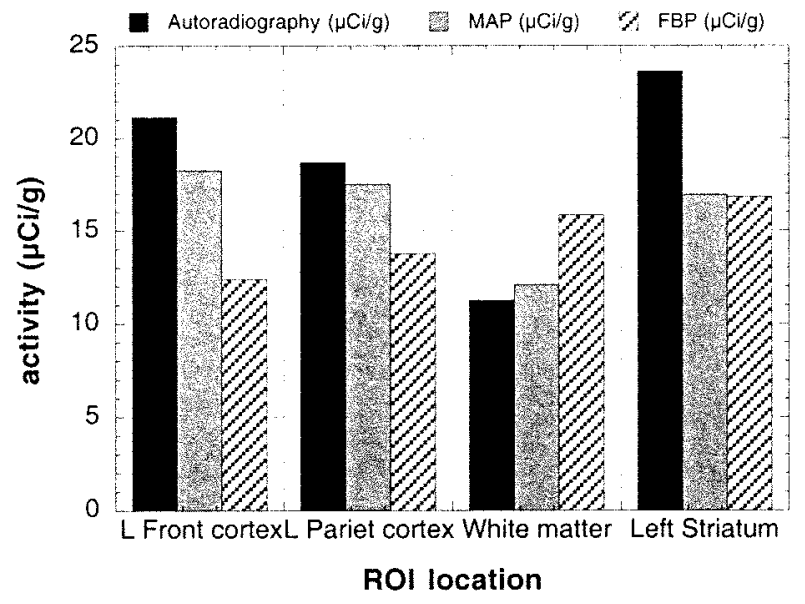

Fig. 10. Absolute ${ }^{18}$ FDG activity concentration as measured in cortical and subcortical structures in the rat brain, with ROI's in the whole head autoradiograph and the PET images.

structure in small animal studies and improves quantification by reducing partial volume and spillover effects. From the study in the monkey brain phantom, it appears that this improvement in resolution can be achieved without a degradation in noise compared with FBP images reconstructed at their highest resolution. The quantification of the MAP algorithm in vivo, as compared with autoradiography, showed significant improvements compared with conventional FBP reconstruction. In addition, the weighting of the smoothing hyperparameter $\beta$ with the mean count level [13] results in a count-independent contrast recovery for the MAP reconstructed images for a specific object. This is verified in the monkey brain phantom ROI's across images reconstructed with counts spanning two orders of magnitude.

A remaining concern is the impact of the spatially variant resolution of the MAP method evaluated here, particularly in dynamic studies where changes in tracer activity will result in time-varying resolution and associated partial volume effects within ROI's. This may in turn produce confounding effects in parameter estimates in kinetic modeling experiments. In this case, the methods described in [14] for compensating for spatially variant resolution through the use of a spatially variant smoothing prior may prove effective.

Given the improvement in resolution and quantitative accuracy seen in vivo with the 3-D MAP algorithm, we anticipate that it will become widely used in PET studies of small animals, in which resolution is often the critical issue. Routine application of a 3-D MAP in quantitative dynamic studies awaits further validation in the effects of isotope redistribution using autoradiography and well counting of tissue samples as a gold standard.

\section{ACKNOWLEDGMENT}

The authors would like to acknowledge valuable assistance from CTI PET Systems Inc., Dr. C. Michel, R. Sumida, J. Edwards, D. Liu, W. Ladno, D. Rubins, and the staff of the UCLA biomedical cyclotron for providing the isotopes. 


\section{REFERENCES}

[1] A. Chatziioannou, S. R. Cherry, Y. Shao, R. W. Silverman, K. Meadors, T. H. Farquhar, M. Pedarsani, and M. E. Phelps, "Performance evaluation of microPET: A high resolution LSO PET scanner for animal imaging," J. Nucl. Med., vol. 40, pp. 1164-1175, 1999.

[2] M. Defrise, P. E. Kinahan, D. W. Townsend, C. Michel, M. Sibomana, and D. F. Newport, "Exact and approximate rebinning algorithms for 3-D PET data," IEEE Trans. Med. Imag., vol. 16, pp. 145-158, 1997.

[3] P. E. Kinahan and J. G. Rogers, "Analytic 3D image reconstruction using all detected events," IEEE Trans. Nucl. Sci., vol. 36, pp. 964-968, 1989.

[4] T. H. Farquhar, A. Chatziioannou, and S. R. Cherry, "An evaluation of exact and approximate 3-D reconstruction algorithms for a high resolution small-animal PET scanner," IEEE Trans. Med. Imag., vol. 17, pp. 1073-1080, 1998.

[5] C. Chen, S. Y. Lee, and Z. H. Cho, "Parallelization of the EM algorithm for 3-D PET image reconstruction," IEEE Trans. Med. Imag., vol. 10, pp. 513-522, 1991.

[6] C. A. Johnson, Y. Yuchen, R. E. Carson, R. L. Martino, and M. E. DaubeWitherspoon, "A system for the 3D reconstruction of retracted-septa PET data using the EM algorithm," IEEE Trans. Nucl. Sci., vol. 42, pp. 1223-1227, 1995.

[7] J. M. Ollinger and A. S. Goggin, "Maximum likelihood reconstruction in fully 3D PET via the SAGE algorithm," presented at the 1996 IEEE Nucl. Sci. Symp., vol. 3, Anaheim, CA, 1996, pp. 1594-1598.

[8] A. Terstegge, S. Weber, H. Herzog, H. W. Muller-Gartner, and H. Halling, "High resolution and better quantification by tube of response modeling in 3D PET reconstruction," presented at the 1996 IEEE Nucl. Sci. Symp., vol. 3, Anaheim, CA, 1996, pp. 1603-1607.

[9] J. Qi, R. Leahy, S. Cherry, A. Chatziioannou, and T. Farquhar, "Highresolution 3D Bayesian image reconstruction using the microPET smallanimal scanner," Physi. Med. Biol., vol. 43, pp. 1001-1013, 1998.

[10] S. R. Cherry, Y. Shao, R. W. Silverman, K. Meadors, S. Siegel, A. Chatziioannou, J. W. Young, W. Jones, J. C. Moyers, D. Newport, A. Boutefnouchet, T. H. Farquhar, M. Andreaco, M. J. Paulus, D. M. Binkley, R. Nutt, and M. E. Phelps, "MicroPET: A high resolution PET scanner for imaging small animals," IEEE Trans. Nucl. Sci., vol. 44, pp. 1161-1166, 1997.
[11] M. Yavuz and J. A. Fessler, "Objective functions for tomographic reconstruction from randoms-precorrected PET scans," presented at the 1996 IEEE Nucl. Sci. Symp., vol. 2, Anaheim, CA, 1996, pp. 1067-1071.

[12] J. A. Fessler and W. L. Rogers, "Spatial resolution properties of penalized-likelihood image reconstruction: Spatial-invariant tomographs," IEEE Trans. Image Procressing, vol. 5, pp. 1346-1358, Sept. 1996.

[13] J. Qi and R. Leahy, "A theoretical study of the contrast recovery and variance of MAP reconstructions from PET data," IEEE Trans. Med. Imag., vol. 18, pp. 293-305, 1999.

[14] _ - "Resolution and noise properties of MAP reconstruction for fully 3D PET," IEEE Trans. Med. Imag., to be published.

[15] E. J. Hoffman, P. Cutler, T. Guerrrero, W. Digby, and J. Mazziotta, "Assesment of accuracy of PET utilizing a 3-D phantom to simulate the activity distribution of 18-F-fluorodeoxyglucose uptake in the human brain,” J. Cerebral Blood Flow Metab., vol. 11, pp. A17-A25, 1991.

[16] C. Riddell, F. Jousse, L. Aloj, P. Mansour, M. Whatley, J. Carrasquillo, M. Daube-Witherspoon, S. Libutti, D. Danforth, and S. Bacharach, "Attenuation correction vs. no attenuation correction: A signal to noise analysis in FDG PET whole body images," J. Nucl. Med., vol. 39, pp. 80P-80P, 1998.

[17] R. Myers, S. Hume, S. Ashworth, A. Lammertsma, P. Bloomfield, S. Rajeswaran, and T. Jones, "Quantification of dopamine receptors and transporter in rat striatum using a small animal PET scanner," in Quantification of Brain Function Using PET, R. Myers, V. Cunningham, D. Bailey, and T. Jones, Eds. San Diego, CA: Academic Press, 1996, pp. 12-15.

[18] S. Siegel and M. Dahlbom, "Implementation and evaluation of a calculated attenuation correction for PET," IEEE Trans. Nucl. Sci., vol. 39, pp. 1117-1121, 1992. 